\title{
Estratégias para 0 enfrentamento das doenças crônicas: um modelo em que todos ganham
}

Strategies for coping with chronic diseases: a model where everyone wins

Resumo

As transformações sociais e econômicas das últimas décadas e suas consequentes alterações nos estilos de vida das sociedades contemporâneas - mudanças dos hábitos alimentares, aumento do sedentarismo e do estresse - e a maior expectativa de vida da população colaboraram para o aumento da incidência das doenças crônicas, que hoje constituem um sério problema de saúde pública. Doenças cardiovasculares, câncer, diabetes e doenças respiratórias são as maiores responsáveis pela mortalidade no mundo. No Brasil, nas últimas décadas, as doenças crônicas não-transmissíveis tornaram-se as principais causas de óbito e incapacidade prematura. Foco criativo deve ser dirigido ao cuidado dos idosos, que são os que mais sofrem os efeitos de sua própria fragilidade e os que mais demandam serviços de saúde. A Agência Nacional de Saúde Suplementar publicou uma resolução que incentiva a participação de beneficiários de planos de saúde em programas de envelhecimento ativo, com a possibilidade de descontos nas mensalidades. Assim, idosos passam a receber benefícios pecuniários para aderir a programas de saúde preventiva e detecção precoce de doenças. Este artigo de opinião visa a apresentar questões relativas não só à recente iniciativa da ANS, mas particularmente da UnATI/UERJ, que ao longo de 18 anos de existência vem trilhando o tema de novos modelos de cuidado. Este texto expressa o mais novo projeto da instituição, que é desenvolver um modelo de linha de cuidados integrando a promoção e a atenção à saúde do idoso.

${ }_{1}$ Universidade Aberta da Terceira Idade-UnATI. Universidade do Estado do Rio de Janeiro. Rio de Janeiro, RJ, Brasil

Correspondência / Correspondence

Renato P. Veras

UnATI / UERJ

Universidade Aberta da Terceira Idade - Direção

Rua São Francisco Xavier, $524-10^{\circ}$ andar, Bloco F - Maracanã

20559-900 Rio de Janeiro, RJ, Brasil

E-mail: unativeras@gmail.com
Palavras-chave:

Envelhecimento.

Promoção de saúde. Saúde

do idoso. Gastos em

saúde. Modelos

assistenciais. Manutenção

da capacidade funcional.

Agência Nacional de

Saúde Suplementar.

UnATI/UERJ. 
Abstract

The social and economic transformations of recent decades and their consequent changes in the lifestyles of contemporary society - changes in eating habits, increased sedentarianism and stress - and longest life expectancy of the population contributed to the increased incidence of chronic diseases, which today are a serious health problem. Cardiovascular diseases, cancer, diabetes and respiratory diseases are the major cause of mortality worldwide. In Brazil, in recent decades, chronic non-communicable diseases have become major causes of premature death and disability. Creative focus should be directed to the care of the elderly, who are the ones who suffer the effects of their own weakness and demand more health services. The National Agency of Supplementary Health published a resolution that encourages the participation of beneficiaries in health insurance programs for active aging, with the possibility of discounts on fees. Thus, the elderly now receive cash benefits to comply with preventive health programs and early detection of diseases. This opinion paper aims to present issues relating not only to the recent initiative of the Agency, but rather to UERJ's Open University for Studies on the Elderly, which for 18 years has been approaching the theme of new health care models. This text expresses the institution's latest project, which is to develop a health care model integrating promotion and attention for the elderly's health.
Key words: Aging. Health expenditures. Health promotions. Health of the Elderly. Health care models. Maintenance of functional capacity; The National Agency of Supplementary Health, UnATI/UERJ.
A principal causa de mortalidade e morbidade no Brasil são as doenças crônicas, que normalmente têm desenvolvimento lento, duram períodos extensos e apresentam efeitos de longo prazo, difíceis de se prever. À semelhança dos demais países ricos, as pesquisas sugerem que as condições complexas, como diabetes e depressão, irão impor uma carga ainda maior no futuro ${ }^{1}$.

Alguns anos atrás, as doenças crônicas eram consideradas um problema de países ricos e de população idosa. Hoje sabemos que dentro de países de alta renda, os pobres, assim como os jovens e as pessoas de meia-idade, são afetados por condições crônicas. As implicações econômicas dessas doenças também são graves: elas têm impacto negativo nos salários, lucros, participação da força de trabalho e produtividade, bem como aumentam a aposentadoria precoce, causando alta rotatividade do emprego e incapacidade. Como a despesa com cuidados com as doenças crônicas sobe em todo o mundo, elas ocupam proporções cada vez maiores nos orçamentos públicos e privados.

As doenças crônicas incluem tradicionalmente as doenças cardiovasculares, diabetes e asma ou doença pulmonar obstrutiva crônica (DPOC).
Como as taxas de sobrevivência e durações têm melhorado, esse tipo de doença agora também passou a incluir muitas variedades de câncer, HIV / Aids, distúrbios psiconeurológicos (como depressão, esquizofrenia e demência) e deficiência, tais como deficiência visual e artroses. A maioria delas não tem cura, mas várias podem ser prevenidas ou controladas por meio da detecção precoce, adoção de dieta e hábitos saudáveis, prática de exercícios e acesso a tratamento adequado.

Muitas condições crônicas estão ligadas a uma sociedade em envelhecimento, mas também às escolhas de estilo de vida, como o tabagismo, consumo de álcool, comportamento sexual, dieta inadequada e inatividade física, além $\mathrm{da}$ predisposição genética. O que elas têm em comum é o fato de precisarem de uma resposta complexa e de longo prazo, coordenada por profissionais de saúde de formações diversas, com acesso aos medicamentos e equipamentos necessários, estendendo-se à assistência social. A maioria dos cuidados de saúde hoje, no entanto, ainda está estruturada em torno de episódios agudos.

Tendo em vista esse cenário, a gestão de doenças crônicas é cada vez mais considerada uma 
questão importante por gestores e pesquisadores em todo o mundo, que buscam intervenções e estratégias para combater esses agravos. A Organização Mundial da Saúde ${ }^{2,3}$ define o gerenciamento de doenças crônicas como a "gestão contínua de condições durante um período de anos ou décadas".

É provável que os gastos em saúde aumentem substancialmente. De fato, os cuidados em saúde tendem a emergir como um dos maiores desafios fiscais nas próximas décadas no Brasil. ${ }^{4}$ Existem duas forças por trás da projeção de aumento desses gastos: a proporção crescente de idosos na população e a intensidade do uso dos serviços de saúde pelos idosos. ${ }^{5}$

Espera-se que três fatores também aumentem o número de idosos que necessitam de cuidados de longo prazo. Primeiro, o forte crescimento do número de pessoas muito idosas, nos próximos 30 anos, resultará em um maior número absoluto de idosos fragilizados, mesmo considerando a redução da proporção da severidade de doenças entre os idosos, devido a avanços na prevenção de doenças e melhores práticas assistenciais. Segundo, a mudança de status das mulheres e dos valores sociais e familiares continuará afetando a disponibilidade de apoio familiar para esses idosos. Projeções para o Brasil estimam que o número de pessoas sendo cuidadas por não-familiares (cuidadores formais) irá duplicar até 2020 e será cinco vezes maior em 2040, em comparação com 2008. ${ }^{6}$ Em terceiro lugar, alguns fatores de risco que alcançavam majoritariamente o homem, sobretudo o consumo do álcool e tabaco, e do estresse no trabalho, passarão também a atingir as mulheres, quando comparados aos números atuais. Uma consequência será a redução da diferença da expectativa de vida, que atualmente no Brasil é oito anos maior para as mulheres.

A organização do sistema de saúde no Brasil precisa ser ajustada para os diferentes perfis demográficos e epidemiológicos decorrentes do aumento da população idosa. A magnitude do aumento dos gastos em saúde com a população idosa dependerá, sobretudo, de esses anos a mais serem saudáveis ou de enfermidades e dependência. A prevenção, a manutenção da saúde, independência e autonomia e o retardamento de doenças e fragilidades em uma população mais velha serão os maiores desafios relacionados à saúde decorrentes do envelhecimento da população. Assim, qualquer política social e de saúde destinada aos idosos deve levar em conta a promoção da saúde e a manutenção da capacidade funcional.

Por um lado, os idosos apresentam maior carga de doenças e incapacidades e usam mais os serviços de saúde; por outro, os modelos vigentes de atenção à saúde do idoso se mostram ineficientes e de alto custo, reclamando estruturas criativas e inovadoras. É por este motivo que, do ponto de vista da saúde pública, a capacidade funcional surge como o conceito de saúde mais adequado para instrumentalizar e operacionalizar uma política contemporânea de atenção à saúde do idoso. Essa política deve, assim, ter como objetivo maior a manutenção da máxima capacidade funcional do indivíduo que envelhece, pelo maior tempo possível.

Há no Brasil um fato novo, em consonância com esta perspectiva de preservar a saúde e propiciar mais anos de vida saudável: a Agência Nacional de Saúde Suplementar (ANS) ${ }^{7}$ publicou em agosto de 2011, no Diário Oficial da União, a Resolução Normativa $n^{\circ} 265$, que incentiva a participação de usuários de planos de saúde em programas de envelhecimento ativo, com a possibilidade de descontos nas mensalidades. ${ }^{8,9}$

Propor que as operadoras de planos de saúde ofereçam benefícios pecuniários aos clientes que participarem de programas de prevenção de doenças e detecção precoce de enfermidades é uma iniciativa bastante oportuna. A proposta da ANS vale para todas as faixas etárias, mas tem impacto particularmente importante para a população da terceira idade, ao estimular ações de envelhecimento ativo. Atualmente, a lógica do sistema de saúde é pautada pelo ciclo de tratamento das doenças, não da prevenção. Inverter esse modelo é imperativo para assegurar mais qualidade de vida aos idosos e bem-estar à população como um todo. 
Se considerarmos os dados demográficos brasileiros, iremos verificar que o país envelhece progressivamente e de forma acelerada. Todo ano, 700 mil novos idosos são incorporados a esse segmento da pirâmide etária - a maior parte com doenças crônicas e alguns com limitações funcionais. Em menos de quatro décadas, passamos de um cenário de mortalidade próprio de uma população jovem para um quadro de enfermidades complexas e onerosas, típicas da terceira idade, caracterizado por doenças que perduram anos, com exigência de cuidados constantes, medicação contínua e exames periódicos. O aumento da expectativa de vida é um fator positivo, mas precisamos ponderar que a longevidade só será de fato uma conquista se agregarmos qualidade aos anos adicionais de vida.

O maior problema da maioria dos modelos assistenciais vigentes talvez seja o foco exclusivo na doença. Mesmo quando oferecem um programa diferenciado, as propostas são voltadas prioritariamente para a redução dos custos de doenças específicas, esquecendo que muitos pacientes possuem múltiplas patologias crônicas. Dados de um estudo americano ${ }^{10}$ mostram inclusive o fracasso de tais programas, pois são realizados numa lógica hospitalocêntrica.

Programas de gerenciamento de doenças para idosos têm uma relação custo-benefício baixa, pois os idosos possuem mais de uma doença crônica, e tratar adequadamente uma doença faz apenas reduzir os índices de morbidade de tal patologia. Como são pacientes que frequentemente possuem múltiplas doenças crônicas e utilizam cuidados de diferentes especialidades médicas, fica evidente que focar apenas uma doença não é a medida mais adequada. A melhor opção é estruturar modelos que funcionem de modo integrado e consigam dar conta de toda a gama de cuidados. Se não for assim, o problema nunca será resolvido, pois as demais doenças e sua fragilidade serão mantidas; além disso, os recursos são utilizados inadequadamente.

Os idosos apresentam maior carga de doenças e incapacidades, e por esse motivo utilizam com mais frequência os serviços de saúde, mas mesmo assim os modelos de cuidado são estruturados à semelhança do adulto jovem. Deste modo, os modelos vigentes de atenção à saúde do idoso se mostram ineficientes e de alto custo. ${ }^{11}$

As conquistas da ciência e da medicina e o vasto conhecimento acumulado deveriam estimular modelos de maior resolutividade e custo mais adequado. No sistema brasileiro, o peso médicoassistencial ainda é preponderante. Planos de saúde operam numa espécie de mutualismo, em que os menos saudáveis consomem mais serviços e inflacionam valores pagos por quem tem capacidade funcional preservada e está em boa forma. A aplicação do aporte epidemiológico e a ênfase em prevenção fazem com que essa nova abordagem favoreça a redução dos custos assistenciais, em paralelo à ampliação da qualidade de vida, o que implica reorganizar os serviços de saúde.

A relação entre prestadoras e clientes de mais idade sempre foi de grande desconfiança. A política das operadoras é tentar reduzir o peso desse segmento em suas carteiras. Os idosos, por seu lado, reclamam do custo, das dificuldades de atendimento e das glosas a muitos dos exames solicitados - não existe outra relação de comércio em que vendedor e clientes têm posições tão antagônicas. Algumas operadoras talvez argumentem que programas de envelhecimento ativo poderão gerar aumento na utilização do plano de saúde; e podem também afirmar que o aumento da sinistralidade, aliado ao desconto concedido no preço e à obrigatoriedade de sua manutenção por 12 meses, seria fator decisivo para recusar a proposta da ANS.

Em relação ao aumento de sinistralidade, a prevenção e a promoção de saúde, se bem aplicadas e avaliadas, podem trazer resultados financeiros positivos no médio e longo prazos. No início da operação, aumenta-se o uso de procedimentos, mas principalmente aqueles de baixo custo. No longo prazo, porém, haverá diminuição dos procedimentos de alto custo e melhoria da saúde. O mais difícil talvez seja a mudança de cultura.

Também observamos enorme abismo entre prática e discurso sobre modelos hierarquizados 
por complexidade que priorizem o cuidado integral para todos os idosos, já que eles têm patologias crônicas e múltiplas. Quando estas surgem (e oito entre dez idosos têm pelo menos uma doença crônica), muito pouco pode ser feito. ${ }^{12}$ Doenças crônicas não regridem, resta apenas conviver com elas. Dessa forma, a ação mais adequada é evitar ou retardar seu aparecimento e desacelerar sua progressão, prolongando o período de vida ativa.

Não é mais cabível manter a lógica da doença. As empresas terão de se adaptar. Com a nova proposta, a ANS faz mais uma tentativa de induzir a lógica preventiva no setor de saúde suplementar. Desta vez, no entanto, a agência lança mecanismos de indução também para os consumidores, cuja adesão é fundamental para o sucesso das ações de prevenção.

As questões apresentadas neste artigo de opinião fazem parte não só da recente resolução da ANS, mas particularmente da UnATI/UERJ, que ao longo dos seus 18 anos de existência vem trilhando o tema de novos modelos de cuidado. ${ }^{12}$ Este texto expressa o mais novo projeto da instituição, que é desenvolver um modelo de linha de cuidados integrando a promoção e a atenção à saúde do idoso.

Pode-se considerar este modelo como uma proposta de aprofundamento de práticas preventivas, balizado pelo afã de detectar precocemente os agravos de saúde em qualquer condição funcional. Sua lógica é estruturada em níveis hierárquicos, nos quais instâncias de cuidado são oferecidas ao idoso de acordo com seu grau de necessidade. Portanto, deve haver o monitoramento constante para se oferecer o cuidado mais adequado com implicação positiva, tanto do ponto de vista clínico como financeiro. A compreensão de que se deve investir no idoso saudável, mesmo aquele com doença crônica e em tratamento" - ou seja, a imensa maioria dos idosos da nossa sociedade -, e ter ações para todos os demais é uma visão contemporânea que os gestores da área deveriam aplicar. A ocorrência de doenças crônicas na população idosa é, sem dúvida, de grande magnitude. Cabe-nos saber, entretanto, o quanto tais patologias os impedem de exercer suas atividades rotineiras de forma independente e autônoma. É a diminuição da capacidade funcional do idoso que o tornará, de alguma forma, dependente de um nível mais complexo de assistência, ou seja, na concepção do modelo que estamos sugerindo, o fará subir para um degrau hierarquicamente mais intenso de cuidados.

Em síntese, um modelo de atenção à saúde do idoso que se pretenda eficiente deve aplicar todos os níveis de cuidado, isto é, possuir um fluxo bem desenhado de ações de educação, promoção da saúde, prevenção de doenças evitáveis, postergação de moléstia, cuidado o mais precocemente possível e reabilitação de agravos. Essa linha de cuidados se inicia na captação, no acolhimento e no monitoramento do idoso e somente se encerra nos momentos finais da vida, na unidade de cuidados paliativos.

Torna-se necessário sempre frisar que, nessa hierarquização da complexidade das fragilidades e agravos, a ênfase é conferida aos níveis básicos, com vistas a ofertar condições de promover um envelhecimento ativo. $\mathrm{Na}$ linha de cuidados que estamos desenvolvendo em sete etapas hierarquizadas de cuidados ascendentes, é nos níveis iniciais que se concentram mais de $90 \%$ da população idosa. 


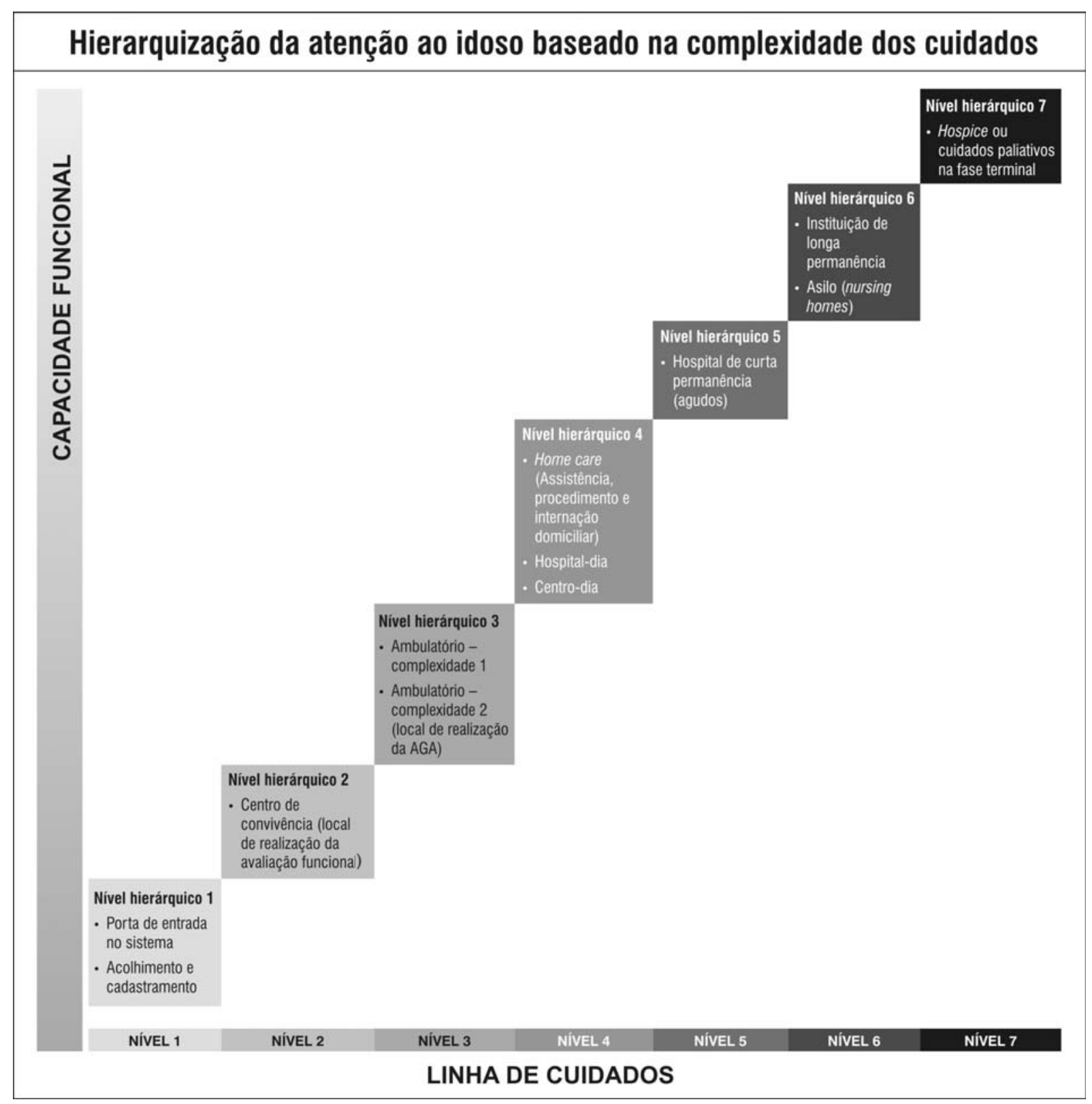

Entendemos esse modelo como uma proposta segundo a qual todos devem ser vencedores: o idoso, que amplia seus anos de vida com qualidade; a família, que terá um ente querido ativo e participativo; e as prestadoras de saúde, à semelhança do SUS, que evitarão internações repetidas e de alto custo.

A atualidade deste debate nos faz lembrar um recente artigo do economista norteamericano Paul Krugman ${ }^{13}$, intitulado "Livre para morrer: reflexão sobre a saúde dos americanos". No texto, Krugman relata que, durante o recente debate das prévias presidenciais do Partido Republicano nos Estados Unidos, ocorrido em setembro de 2011, Wolf Blitzer, apresentador da emissora de televisão CNN, perguntou ao deputado Ron Paul o que se deveria fazer se um homem de 30 anos, que optou por não comprar um plano de saúde, se visse de repente precisando de seis meses de terapia intensiva. Paul respondeu: "Liberdade envolve isso: assumir seu próprio risco". Blitzer pressionou de novo, perguntando se "a sociedade deve simplesmente deixá-lo morrer?”. E a plateia explodiu em vivas e gritos de "sim!". 
O incidente acentuou algo muito estranho para nós brasileiros, país em que todos os cidadãos têm sua cobertura de saúde garantida na Constituição, além de existir o setor privado de saúde como opção suplementar. No país mais rico e poderoso do mundo, pessoas que não podem pagar o atendimento médico frequentemente ficam sem ele - e às vezes morrem em consequência disso. $\mathrm{Na}$ verdade, um segmento conservador $\mathrm{da}$ sociedade americana se dispõe a deixar que as pessoas sem seguro-saúde, mesmo que não seja por sua culpa, morram por falta de atendimento? A resposta, com base na história recente, é um sonoro "sim!".

Este fato, ocorrido recentemente (em setembro de 2011), nos mostra algumas verdades: primeiro, que muitos consideram saúde como um produto que, com o aumento dos custos, deve ser restrito e não extensivo a todos. Esta visão, mesmo não sendo hegemônica, nos leva a um alerta. Os custos da saúde não param de crescer, mas as fontes de financiamento são finitas. Deste modo, ao pensarmos em ações de promoção e prevenção para uma sociedade que envelhece rapidamente, como é o caso do Brasil, o que está embutido nesta reflexão não é apenas o desejo de se oferecer mais anos de vida saudável à população, mas também um movimento que visa a consolidar a importância de ações eficientes que permitam ao setor Saúde, particularmente o SUS, ter condições de ofertar atendimento a todos.

Até bem pouco tempo atrás, dinheiro era algo que passava longe dos modelos de cuidado na área da saúde; hoje, não falar de financiamento é colocar em risco todo o sistema - e o que é mais grave, compactuar com mortes de indivíduos, não por estarem doentes, mas pelo simples fato de não possuírem condições de financiar seu cuidado.

As transferências públicas no Brasil têm sido muito eficazes para reduzir a pobreza e a desigualdade, em particular para a maioria da população mais velha. No entanto, isso foi alcançado a um custo elevado. Como desejamos a manutenção e ampliação desta prosperidade, e sabemos que o envelhecimento populacional continuará a pressionar os sistemas sociais, ao defender modelos de saúde que tenham foco na prevenção e no monitoramento ao longo do curso de vida, estamos expressando que sabemos que as definições no momento atual são escolhas críticas, com consequências particularmente cruciais para os grupos vulneráveis e para a perspectiva de crescimento do país.

Esta dimensão ampla é fundamental para realçar de modo categórico que, para se tornar factível e para que haja saúde para todos com qualidade, quer no setor público, quer no privado, é necessário mudar a lógica do modelo do período pregresso, quando as doenças eram agudas e a população morria cedo. O Brasil mudou, e os tempos são outros. A transição epidemiológica ocorreu e o atual padrão de doença é majoritariamente de doenças crônicas nãotransmissíveis, do mesmo modo que ocorreu a transição demográfica e esta demonstra que o Brasil é um país jovem de cabelos brancos. ${ }^{14}$ 


\section{REFERÊNCIAS}

1. Moraes EM, Marino MCA, Santos RR. Principais síndromes geriátricas. Rev Med Minas Gerais 2010; 20(1): 54-6.

2. Organização Mundial da Saúde (OMS). Grupo de Doenças Não-transmissíveis e Saúde Mental. Departamento de Prevenção de Doenças Nãotransmissíveis Promoção de Saúde.

Envelhecimento ativo: um marco para elaboração de políticas. 2001 [ Acesso em 18 jan 2011].Disponível em: http://www.unati.uerj.br/ destaque/Madri.doc.

3. World Health Organization. Envelhecimento ativo: uma política de saúde. Brasília: OPAS; 2005.

4. Banco Mundial. Population aging: is Latin America ready? Washington: The World Bank; 2011.

5. National Institute of Aging. National Institutes of Health, US Dept. Why population aging matters: a global perspective. Washington : NIA; 2007.

6. Banco Mundial. Envelhecendo em um Brasil mais velho: implicações do envelhecimento populacional para o crescimento econômico, a redução da pobreza, as finanças públicas e a prestação de serviços. Washington: The World Bank; 2011.

7. Ministério da Saúde (Brasil). Agência Nacional de Saúde(ANS) . Resolução n ${ }^{\circ}$ 265, de 19 de
Agosto de 2011. Suplementar. Diário Oficial da União 20 ago 2011. [ Acesso em 03 out 2011].

8. Veras RP. Uma saída para a crise no setor saúde. Rev Idec 2011; 157: 35.

9. Veras RP. Experiências e tendências internacionais de modelos de cuidado para com o idoso. Rev Ciênc Saúd Colet 2011. [ Acesso em 03 out 2011] Disponível em : http:// www.cienciaesaudecoletiva.com.br/artigos/ artigo_int.php?id_artigo $=8612$.

10. Agency for Healthcare Research and Quality. The high concentration of U.S. health care expenditures. Rockville AHRQ.2005; 163(19):2359-67

11. Veras RP. Envelhecimento populacional contemporâneo: demandas, desafios e inovações. Rev Saúd Pública 2009; 43(3): 548-54

12. Veras RP, Caldas CP. UnATI-UERJ: 10 anos um modelo de cuidado integral para a população que envelhece. Rio de Janeiro: UnATI; 2004.

13. Krugman P. Free to Die: The New York Times. 2011 [ Acesso em 03 jan 2011] Disponível em: http://www.nytimes.com/2011/09/16/opinion/ krugman-free-to-die.html?_r=2\&hp.

14. Veras RP. País jovem com cabelos brancos: a saúde do idoso no Brasil. Rio de Janeiro: Relume-Dumará; 1994. 